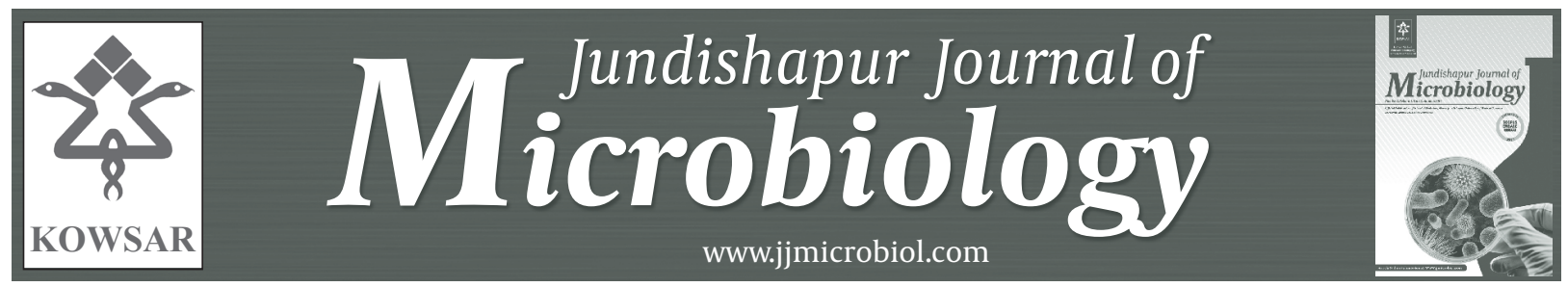

\title{
Comparison the Sensitivity of Stomach Mucus Touching Cytology and Urease Rapid Test in Helicobacter Pylori Diagnosis in Endoscopies Patients with Gastritis or Peptic Ulcer
}

\author{
Mohammad Jafari ${ }^{1,}$, Alireza Khalilian ${ }^{2}$, Farshad Rostampour ${ }^{3}$ \\ ${ }^{1}$ Department of Pathology, Faculty of Medicine, Hamadan University of Medical Sciences and Health Services, Hamadan, IR Iran \\ ${ }^{2}$ Department of Internal Medicine, Faculty of Medicine, Hamadan University of Medical Sciences and Health Services, Hamadan, IR Iran \\ ${ }^{3}$ Students' Research Committee (SRC), Faculty of Medicine, Hamadan University of Medical Sciences and Health Services, Hamadan, IR Iran \\ *Corresponding author: Mohammad Jafari, Department of Pathology, Faculty of Medicine, Hamadan University of Medical Sciences and Health Services, \\ Hamadan, IR Iran. Tel:+918-8118991, E-mail:mj105105@yahoo.com.
}

Keywords: Helicobacter Pylori; Gastritis

Article type: Letter To Editor; Received: 25 Jul 2012; Revised: 08 Dec 2012; Accepted: 22 Dec 2012; Epub: 01 Jun 2013; Ppub: Jun 2013

Implication for health policy/practice/research/medical education:

Due to gained results from this study, it is proposed to use of stomach mucus touching cytology as the first step of early diagnosis of $\mathrm{H}$. pylori among inpatients in hospitals. It can be also used for outpatients because it has the same sensitivity as histology and also it is simple, fast, cheap and available and can prevent the problems of no treatment and wasting time and money for $\mathrm{H}$. pylori diagnosis. It is proposed to do this study in outpatients to gain more reliable statistics by comparing the stomach mucus touching cytology and urease rapid test

>Please cite this paper as:

Jafari M, Khalilian A, Rostampour F. Comparison the Sensitivity of Stomach Mucus Touching Cytology and Urea's Rapid Test in Helicobacter Pylori Diagnosis in Endoscopies Patients with Gastritis or Peptic Ulcer. Jundishapur J Microbiol. 2013;6(4):e7446. DOI: $10.5812 / \mathrm{jjm} .7446$

Copyright @ C 2013, Ahvaz Jundishapur University of Medical Sciences; Published by Kowsar Corp.

This is an Open Access article distributed under the terms of the Creative Commons Attribution License (http://creativecommons.org/licenses/by/3.0), which permits unrestricted use, distribution, and reproduction in any medium, provided the original work is properly cited. 


\section{Dear Editor,}

Helicobacter Pylori (H. pylori) is microaerophile bacterium that colonizes the human gastrointestinal tract. It is found in the deepest parts of mucus gel that covers stomach mucus or between mucus layer and stomach epithelium $(1,2)$. H. pylori is one of the proved causes of some prevalent diseases like different kinds of gastritis (chronic, acute, atrophic ), peptic ulcer and also dangerous diseases like stomach lymphoma and adenocarcinoma and estimated to be responsible for approximately $65 \%$ of all stomach cancers worldwide (3-5).

There are different diagnosis methods for H. pylori that the most spread ones are: histology of stomach biopsy sample, smear assessment of stomach biopsy sample, culture, serum assessment of bacteria antibodies, urease rapid test, urea breathing test and assessment of discharged antibodies of bacteria. Among these methods; histology of stomach biopsy sample is the most characteristic and sensitive one which is called "Golden Standard". But it is expensive and its results reveal after a few days $(1,2)$. Stomach mucus touching cytology and urease rapid test are adequately characteristic and sensitive and also fast, inexpensive and available $(1,2,6)$.

As present studies report that the specificity of stomach mucus touching cytology and urease rapid test are the same (approximately 100\%) and due to the importance of the assessment of these two tests sensitivity; we decided to assess their sensitivity (6-10)

As present studies report that the specificity of stomach mucus touching cytology and urease rapid test are the same (approximately 100\%) and due to the importance of the assessment of these two tests sensitivity; we decided to assess their sensitivity (6-10). The main aim of our study is a Comparison between the sensitivity of stomach mucus touching cytology, urease rapid test and histology in $H$. pylori diagnosis in endoscoped patients in Ekbatan Hospital of Hamadan.

The present study is an analytic comparative research and sample gathering is futuristic. Samples volume was 120 cases ( appointing the sample volume upon statistic formula was not possible because of the difference between the diagnosis value of stomach mucus touching cytology in previous studies $(6,10,11)$. Thus, 120 cases were chosen according to the similar studies and practical problems. Statistic population was the endoscoped inpatients with gastritis or peptic ulcer in Ekbatan Hospital of Hamadan in one year.

To do this study; two biopsy samples were taken from stomach entre of the patients with gastritis or peptic ulcer. One of the samples was sent for stomach mucus touching cytology (sign of pressed sample on the slide) and urease rapid test, and the other sample was sent for pathology. The taken sample of stomach mucus touching cytology was firstly put on a slide and lightly touches an alcohol-clean class slide repeatedly in serial adjacent area with the cut surface of the tissue. As each slide is pre- pared, dry it rapidly by waving it in the air. For standardization purposes, fix (after drying) in methyl alcohol, and then both slides were fixed by alcohol $90 \%$ and were put in the slide basket.

At the end of each day; all fixed slides were sent to the lab for Giemsa staining. After staining they were assessed for $H$. pylori under the microscope by a pathologist. After stomach mucus touching cytology, the sample on the first slide, was put in the urease rapid and solid kit by a sterile needle. Test results were assessed after 24 hours. The second biopsy sample was dropped into the biopsy jar filled with formalin and was sent to the pathology section. The gained results of these two methods - stomach mucus touching cytology and urea rapid test-were filled in a questionnaire and were compared with histology as a "Gold Standard". Assessment of histology and stomach mucus touching cytology was done by the same pathologist.

Among 120 under study patients;72 persons were men (60\%) and 48 were women (40\%). $19.16 \%$ of these patients were between $17-40$ years old. $31.66 \%$ were between 40 60 and $45.83 \%$ were between $60-80$ and $3.33 \%$ were over 80 years. The average age was $57 / 58 \pm 17$ and they were between 17-87 years old. Among these patients; 92 persons (76.7\%) had gastritis and 28 patients (23.3\%) had peptic ulcer. Among these patients; 83 persons (69.17\%) had $H$. pylori, from which; 60 patients $(72.29 \%)$ had gastritis and 23 patients $(27.71 \%)$ had peptic ulcer. Sensitivity of urease rapid test for $H$. pylori diagnosis was $31.33 \%$.

Sensitivity of stomach mucus touching cytology in this study was $100 \%$ (Table 1). In the patients with gastritis sensitivity of urease rapid test for $H$. pylori diagnosis was $33.33 \%$ and sensitivity of stomach mucus touching cytology was $100 \%$. In the patients with peptic ulcer; sensitivity of the urease rapid test for $H$. pylori diagnosis was $26.08 \%$ and sensitivity of stomach mucus touching cytology was $100 \%$ (Table 2). There was no false positive case in Stomach mucus touching cytology and urease rapid test in this study.

Table 1. Comparison of Stomach Mucus Touching Cytology and Urease Rapid Test to Matching Histology in H. pylori Detection

\begin{tabular}{llll}
\hline $\begin{array}{l}\text { Stomach Mucus } \\
\text { Cytology }\end{array}$ & \multicolumn{3}{c}{ Histology } \\
\hline & Positive & Negative & Total \\
\hline Positive & 83 & 0 & 83 \\
Negative & 0 & 37 & 37 \\
Total & 83 & 37 & 120 \\
Ureaserapid test & & & \\
Positive & 26 & 0 & 26 \\
Negative & 57 & 37 & 94 \\
Total & 83 & 37 & 120 \\
\hline
\end{tabular}


Table 2. Comparison Sensitivity of Stomach Mucus Touching Cytology and Urease Rapid Test in H. pylori Diagnosis in Patients with Gastritis or Peptic Ulcer

\begin{tabular}{lll}
\hline & Sensitivity of Urease Rapid Test, \% & $\begin{array}{l}\text { Sensitivity of Stomach Mucus Touch- } \\
\text { ing Cytology, \% }\end{array}$ \\
\hline Gastritis & 33.33 & 100 \\
\hline Peptic ulcer & 26.08 & 100 \\
\hline All patients (Gastritis and peptic ulcer) & 31.33 & 100 \\
\hline
\end{tabular}

In this study we compare sensitivity of stomach mucus touching cytology and urea's rapid test in diagnosis of $H$. pylori in endoscoped patients. In our study sensitivity of stomach mucus touching cytology was $100 \%$ which is very high and equal to histology as a gold standard method in diagnosis of $H$. pylori. The high sensitivity of stomach mucus touching cytology in $H$. pylori diagnosis was the same as in previous studies. Misra et al. (12) report that the sensitivity of stomach mucus touching cytology was equal to the histology (100\%), which was taken as the gold standard. In study of Trevisani et al. (9) reported that stomach mucus touching cytology has sensitivity of $100 \%$.

That both of these studies have similar results to our investigation. But in another study, Rahbar et al. (13) report that the sensitivity of stomach mucus touching cytology was $96.6 \%$. Yasar et al. (14) report the sensitivity of stomach mucus touching cytology was $93.3 \%$. This difference can be explained by higher sample size in these studies in comparison to our investigation. Biopsy of different part of the stomach could be another cause, we use of the biopsies from the entry of stomach but Rahbar et al. and Yashar et al. use of antral biopsies. Low sensitivity of urease rapid test was also the same as previous studies (10, 11).

Due to this study on inpatients and possibility of presence of some destroyable conditions for urease rapid test, like using of protein pomp inhibitors (PPI) bismuth and antibiotics, and gastric hemorrhage, that cause false negative in rapid urease test (RUT) and decrease the test sensitivity (2), and considering the high sensitivity of stomach mucus touching cytology and its big difference with the sensitivity of urease rapid test, it is concluded that stomach mucus touching cytology is the best method for $H$. pylori diagnosis among inpatients. Otherwise, considering stomach mucus touching cytology as a simple, fast, cheap and available test for $H$. pylori diagnosis $(7,10,11)$; it can be easily taken place of urease rapid test among inpatients.

Due to gained results from this study, it is proposed to use of stomach mucus touching cytology as the first step of early diagnosis of $H$. pylori among inpatients in hospitals. It can be also used for outpatients because it has the same sensitivity as histology and also it is simple, fast, cheap and available and can prevent the problems of no treatment and wasting time and money for $H$. pylori diagnosis.

It is proposed to do this study in outpatients to gain more reliable statistics by comparing the stomach mucus touching cytology and urease rapid test. It is also advised to do other studies with more cases and comparing other H. pylori diagnosis tests like urea breathing test and serology methods with stomach mucus touching cytology.

\section{Acknowledgements}

This research was supported by Vice Chancellor of Research of Hamadan University of Medical Sciences.

\section{Financial Disclosure}

None declared.

\section{Funding/Support}

None declared.

\section{Author Contribution}

None declared.

\section{References}

1. Longo DL, Kasper DL, Jameson JL, Fauci AS, Hauser SL, Loscalzo J. Harrison' ${ }^{\mathrm{TM}}$ PRINCIPLES OF INTERNAL MEDICINE. 2012.

2. Suzuki N, Yoneda M, Naito T, Iwamoto T, Masuo Y, Yamada K, et al. Detection of Helicobacter Pylori DNA in the saliva of patients complaining of halitosis. J Med Microbiol. 2008;57(Pt 12):1553-9.

3. Abe Y, Ohara S, Koike T, Sekine H, Iijima K, Kawamura M, et al. The prevalence of Helicobacter Pylori infection and the status of gastric acid secretion in patients with Barrett's esophagus in Japan. Am J Gastroenterol. 2004;99(7):1213-21.

4. Parkin DM. The global health burden of infection-associated cancers in the year 2002. Int J Cancer. 2006;118(12):3030-44.

5. Ricci C, Vakil N, Rugge M, Gatta L, Perna F, Osborn JF, et al. Serological markers for gastric atrophy in asymptomatic patients infected with Helicobacter Pylori. American J Gastroenterol. 2004;99(10):1910-1915.

6. Pasechnikov VD, Chukov SZ, Kotelevets SM, Mostovov AN, Mernova VP, Polyakova MB. Possibility of non-invasive diagnosis of gastric mucosal precancerous changes. World J Gastroenterol. 2004;10(21):3146-3150.

7. Hino B, Eliakim R, Levine A, Sprecher H, Berkowitz D, Hartman $\mathrm{C}$, et al. Comparison of invasive and non-invasive tests diagnosis and monitoring of Helicobacter Pylori infection in children. JPediatr Gastroenterol Nutr. 2004;39(5):519-23.

8. Tokunaga Y, Shirahase H, Yamamoto E, Inao R, Hamaguchi S, Kanaji K, et al. Modified rapid urease test for Helicobacter Pylor detection in relation to an immunohistochemical stain.J Gastroenterol Hepatol. 2000;15(6):617-21.

9. Trevisani L, Sartori S, Ruina M, Caselli M, Abbasciano V, Grandi E, et al. Touch cytology. A reliable and cost-effective method for diagnosis of Helicobacter Pylori infection. Dig Dis Sci. 1997;42(11):2299303. 
10. Yamamoto T. [Evaluation of usefulness of touch smear cytology for the diagnosis of Helicobacter Pylori infection]. Kansenshogaku Zasshi. 2001;75(10):856-62.

11. Castro Fernandez M, Sanchez Munoz D, Garcia Diaz E, Galan Jurado MV, Rodriguez Alonso C. Diagnosis of Helicobacter Pylori infec tion using urease rapid test in patients with bleeding duodenal ulcer: influence of endoscopic signs and simultaneous corporal and antral biopsies. Rev Esp Enferm Dig. 2004;96(9):599-602.

12. Misra SP, Dwivedi M, Misra V, Gupta SC. Imprint cytology--a cheap, rapid and effective method for diagnosing Helicobacter Pylori. Postgrad Med J1993;69(810):291-5.

13. Rahbar M, Mardanpur K, Tavafzadeh R. Imprint cytology: a simple, cost effectiveness analysis for diagnosing Helicobacter Pylori, in west of Iran. Med J Islamic Republic Iran 2012;26(1):12-6.

14. Yașar N, Muharrem B, İlyas ÖH, Nevin Y. Imprint cytology: A cheap effective method fordiagnosing Helicobacter Pylori. Turk J Gastroenterol 2000;11(1):30-3. 